\title{
Durchatmen? Fehlanzeige! Das akute Lungenversagen (ARDS)
}

Michael Gores, Jennifer Schneck

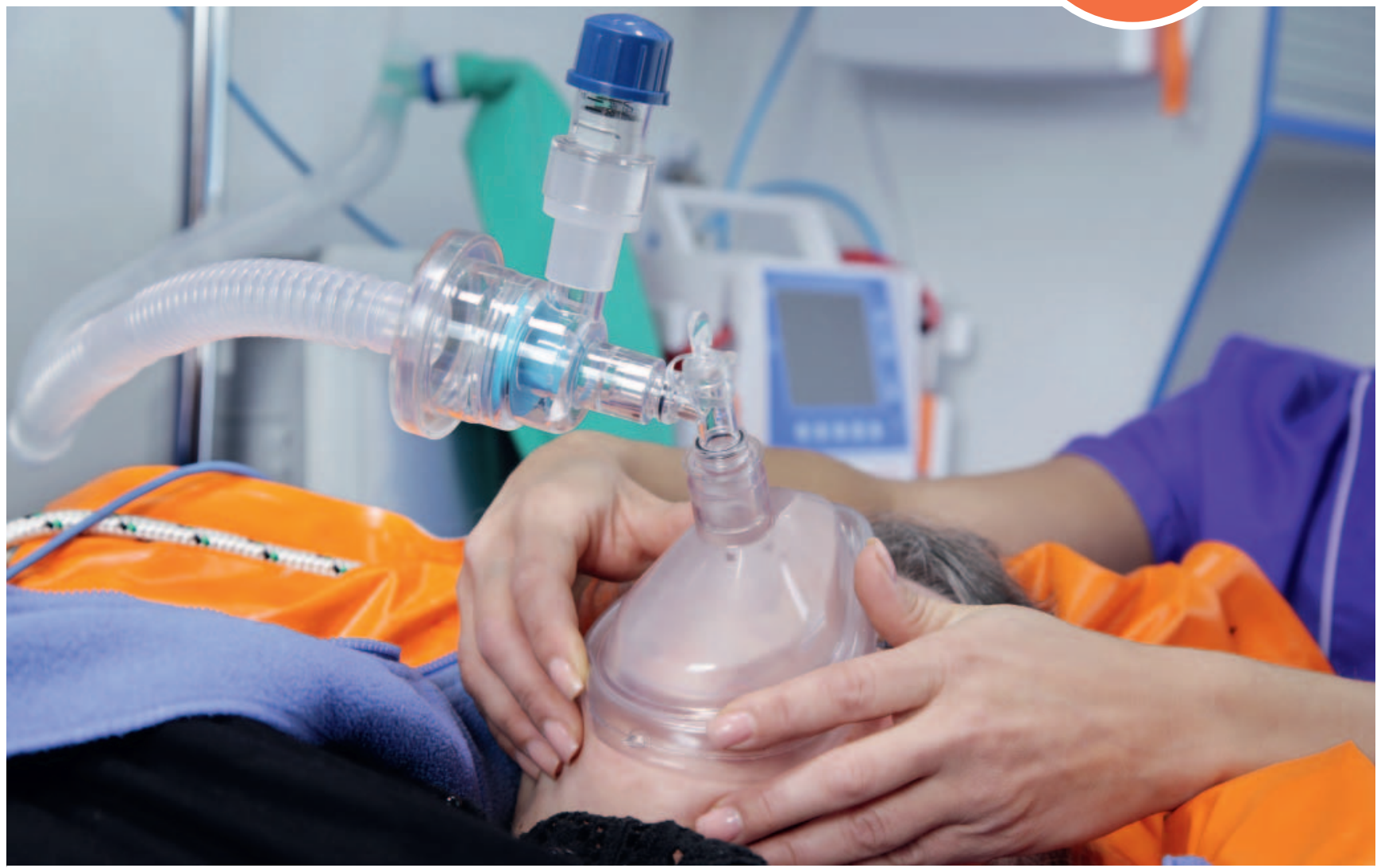

Quelle: vera7388/AdobeStock.com (Symbolbild)

Das akute Lungenversagen ist eine lebensbedrohliche Erkrankung, die mit einer Sterblichkeit von $>40 \%$ der Patienten beschrieben wird. Das frühe Erkennen und der zeitnahe Beginn einer spezifischen Therapie sowie supportiver Maßnahmen können die Letalität entscheidend senken. In diesem Beitrag wird ein Überblick von der Geschichte bis hin zu den aktuellen Behandlungsempfehlungen gegeben.

\section{FALLBEISPIEL}

Alarmierung: Notfallverlegung/ARDS, ECMO. Nach dem Eintreffen in der Klinik werden Sie über die Situation informiert. 26-jährige Patientin mit Darmperforation und Peritonitis. Nach problemloser Extubation klagt die Patientin am 1. postoperativen Tag über Hustenreiz und beginnende Atemnot. Eine Sauerstoffgabe sowie eine nichtinvasive Atemtherapie (NIV) erzielen keine Verbesserung der Sauerstoffsättigung $\left(\mathrm{SpO}_{2}\right)$. Es erfolgt eine erneute Intubation und Lagerung der Patientin auf den Bauch. Bedingt durch eine weiter andauernde Hypoxämie erfolgt im Verlauf die Hinzuziehung des ECMO-Teams (Extra-Corporale MembranOxygenierung). Zusammen mit dem ECMO-Team transportieren Sie die Patientin in die nächste Uniklinik.

\section{Historischer Rückblick}

Es ist der Scham des französischen Arztes René Laënnec zu verdanken, dass wir heute ein Stethoskop zur Verfü- 
- Tab. 1 Beispiele für eine indirekte/direkte Lungenschädigung als Ursache eines ARDS.

\begin{tabular}{|l|l|}
\hline indirekte Schädigung der Lunge & direkte Schädigung der Lunge \\
\hline Schweres Trauma inkl. Verbrennung & Pneumonie \\
\hline Massivtransfusion & Trauma (Lungenkontusion) \\
\hline Pankreatitis & Aspiration \\
\hline Sepsis (nicht von der Lunge ausgehend) & Inhalation toxischer Substanzen \\
\hline
\end{tabular}

gung haben. Als er im Jahre 1816 einer jungen Frau mit vermutetem Herzleiden sein Ohr nicht auf die Brust legen wollte, rollte er ein Papierheft zusammen und benutzte dieses als improvisiertes Hörrohr [1]. Damit ließen sich die Geräusche aus dem Brustkorb sogar besser darstellen, wie er feststellte. Ihm wird auch die Prägung des Begriffs Stethoskop: stethos (Brustkorb) und skopeĩn (betrachten/beschauen) zugeschrieben. 1821 beschrieb Laënnec in seinem Werk „A Treatise on Diseases of the Chest" ein Lungenödem, das sich nicht durch einen Herzfehler erklären ließ [2].

Bis zur ersten Verwendung des Terminus „ARDS (Acute Respiratory Distress Syndrom)“ im Jahre 1967 durch Ashbaugh et al. wurden verschiedene Begriffe für diese Art von Lungenödem verwendet (z. B. „wet lung“, „shock lung“). Ashbaugh und Petty behandelten auf ihren Intensivstationen 272 Patienten, von denen 12 Patienten ein massives Lungenversagen entwickelten, das deutliche Ähnlichkeiten zum Atemnotsyndrom bei Kindern aufwies. Die Erkenntnisse aus diesen Patienten wurden 1967 unter dem Titel „Acute respiratory distress in adults" in Lancet [3, 4] veröffentlicht. Erst viele Jahre später, 1994, wurde durch die Amerikanisch-Europäische Konsensuskonferenz eine erste einheitliche Definition veröffentlicht, die mit der Berlin-Klassifikation 2012 die derzeit letzte Aktualisierung fand $[5,6]$.

$\begin{array}{ll}\text { ABKÜRZUNGEN } \\ \mathrm{ALI} & \text { acute lung injury } \\ \mathrm{ARDS} & \text { Acute Respiratory Distress Syndrome } \\ \mathrm{ECMO} & \text { Extra-Corporal Membrane Oxygenation } \\ \mathrm{FiO}_{2} & \text { Fraction of Inspired Oxygen } \\ \mathrm{IBW} & \text { Ideal Body Weight } \\ \mathrm{kg} \mathrm{KG} & \text { Kilogramm Körpergewicht } \\ \mathrm{NIV} & \text { Non-Invasive Ventilation } \\ \mathrm{paO}_{2} & \text { Sauerstoffpartialdruck } \\ \mathrm{PEEP} & \text { Positive End-Exspiratory Pressure } \\ \mathrm{SpO}_{2} & \text { Sauerstoffsättigung pulsoximetrisch } \\ & \text { gemessen }\end{array}$

\section{Klinik und Pathophysiologie}

Bei einem ARDS kommt es innerhalb von Stunden bis zu einer Woche nach einem auslösenden Ereignis zu einer schweren Gasaustauschstörung. Patienten weisen eine erschwerte Atmung (Dyspnoe), schnellere Atmung (Tachypnoe) und eine blaurote Verfärbung der Haut bzw. Schleimhäute (Zyanose) auf. Im Röntgenbild lassen sich zudem in beiden Lungen Infiltrate nachweisen. Bei den auslösenden Ereignissen wird zwischen einer direkten und einer indirekten Lungenschädigung unterschieden $(\triangleright$ Tab. 1).

Während der Atmung gelangt Sauerstoff von der Alveole über Diffusion ins Blut. Wie im Fickschen Diffusionsgesetz (Adolf Fick 1829-1901) beschrieben, ist die Nettodiffusion (J) abhängig vom Diffusionskoeffizienten (D) des Gases, der Größe der Austauschfläche (A), dem Konzentrationsunterschied $(d C)$ zwischen den Austauschräumen sowie der Diffusionsstrecke (d). Im Rahmen einer Lungenschädigung werden im Körper inflammatorische Prozesse aktiviert, die zu einer Ausschüttung von Zytokinen (z. B. Interleukine, Interferone) führen. Diese Entzündungsreaktion betrifft vor allem die Alveolen sowie das Kapillarendothel und führt zu einer erhöhten Permeabilität.

\section{FORMEL \\ Ficksches Diffusionsgesetz $J=D \times A \times d C / d$}

J: Nettodiffusion; $D$ : Diffusionskoeffizient;

$A$ : Austauschfläche; $d C$ : Konzentrationsunterschied; d: Diffusionsstrecke

Durch das massive Lungenödem wird die Diffusionsstrecke (d) deutlich vergrößert. Eine Störung der Pneumozyten Typ II in der Lunge und die damit verbundene verminderte Bildung des Surfactants (Surface active agent) führt zu einem Kollaps der Alveolen (Atelektasenbildung). Mikrothromben, die durch eine Aktivierung der Gerinnungskaskade entstehen, bewirken eine pulmonal-arterielle Hypertonie und Rechtsherzbelastung. Ein abgeschwächter Euler-Liljestrand-Mechanismus, bei dem schlecht belüftete Lungenareale infolge einer Vasokonstriktion vermindert durchblutet werden, sorgt für Erhöhung des Rechts-LinksShunts. Dabei gelangt sauerstoffarmes Blut zum linken Herzen. Atelektasen, Mikrothromben und ein erhöhter Rechts-Links-Shunt reduzieren die Austauschfläche (A). Gemäß Diffusionsgesetz wird durch eine Verminderung der Austauschfläche $(A)$ und die Erhöhung der Diffusionstrecke $(d)$, die Nettodiffusion $(J)$ massiv reduziert. Der in der Blutgasanalyse gemessene Sauerstoffpartialdruck $\left(\mathrm{p}_{\mathrm{a}} \mathrm{O}_{2}\right)$ sinkt ab und erreicht im Verlauf kritische Werte. 


\section{Einteilung}

Eine Möglichkeit, den Gasaustausch zu beurteilen, bietet der Oxygenierungsindex (Horowitz-Index). Durch Division des aktuellen Sauerstoffgehalts $\left(\mathrm{p}_{\mathrm{a}} \mathrm{O}_{2}\right)$ im Blut mit der inspiratorischen Sauerstoffkonzentration $\left(\mathrm{F}_{\mathrm{i}} \mathrm{O}_{2}=\right.$ fraction of inspired oxygen) erhält man einen Wert, der eine gute Verlaufsbeurteilung erlaubt. Während ein lungengesunder Patient einen maximalen Wert von $500 \mathrm{mmHg}$ erreicht, sinkt der Wert je nach Schweregrad des ARDS ab.

\section{FORMEL}

Oxygenierungsindex bzw. Horowitz Index

$$
\text { Oxygenierungsindex }(\mathrm{OI})=\frac{\mathrm{paO}_{2}(\mathrm{mmHg})}{\mathrm{F}_{\mathrm{i}} \mathrm{O}_{2}}
$$

Schon 1994 wurde dieser Index genutzt, um die Schwere des Lungenversagens zunächst in 2 Grade einzuteilen. Aufgrund neuer Erkenntnisse erarbeitete eine Expertengruppe in Berlin („Berlin-Klassifikation“) im Jahr 2012 eine neue Einteilung ( $\triangleright$ Tab. 2). Die wichtigste Veränderung ist der Verzicht auf den Begriff „Acute Lung Injury“ (ALI) zugunsten einer Einteilung in 3 Schweregrade. Der Oxygenierungsindex wird dabei zwingend bei einem positiven endexspiratorischen Druck (PEEP, positive endexspiratory pressure) gemessen [7].

\section{Erkennung}

Das Erkennen eines Patienten mit ARDS gilt als der erste und somit auch wichtigste Schritt zur Einleitung einer leitliniengerechten Therapie.

\section{Therapiestrategie}

Zwei Behandlungsansätze werden zeitgleich verfolgt. Zum einen die kausale Behandlung der auslösenden Ursache (z. B. antibiotische Therapie einer Pneumonie bzw. chirurgische Infektionsherdsanierung) und zum anderen supportive Maßnahmen zur Verbesserung des Gasaustausches. Sobald der Verdacht eines ARDS im Raum steht, sind die Kriterien anhand der zurzeit gültigen Berlin-Klassifikation zu prüfen, und die entsprechende Diagnostik ist durchzuführen. Bei Erfüllung der Kriterien erfolgt neben den Basismaßnahmen die weitere Behandlung je nach Schweregrad, wie im Flussdiagramm dargestellt ( $\triangleright$ Abb. 1).

\section{Beatmung}

Die maschinelle Beatmung ist eine der essenziellen therapeutischen Maßnahmen in der konservativen Behandlung, birgt jedoch auch die Gefahr, selbst lungenschädigend zu wirken. Als Faktoren, die eine beatmungsinduzierte Schä-
- Tab. 2 Einteilung des ARDS nach alter und aktueller Definition.

\begin{tabular}{|c|c|}
\hline $\begin{array}{l}\text { Amerikanisch-Europäische } \\
\text { Konsensuskonferenz } 1994\end{array}$ & Berlin-Klassifikation 2012 \\
\hline $\begin{array}{l}\text { bilaterale diffuse Infiltrate im } \\
\text { Röntgenbild }\end{array}$ & $\begin{array}{l}\text { bilaterale diffuse Infiltrate im } \\
\text { Röntgenbild }\end{array}$ \\
\hline akuter Beginn & Beginn innerhalb einer Woche \\
\hline $\begin{array}{l}\text { Ausschluss einer Druckerhöhung im } \\
\text { linken Vorhof des Herzens (Pulmo- } \\
\text { naliskatheter) }\end{array}$ & $\begin{array}{l}\text { Ausschluss einer kardialen Ursache } \\
\text { und Volumenüberladung }\end{array}$ \\
\hline $\begin{array}{l}\text { ALI: } \mathrm{p}_{\mathrm{a}} \mathrm{O}_{2} / \mathrm{F}_{\mathrm{i}} \mathrm{O}_{2} \text {-Quotient } \\
<300 \mathrm{mmHg}\end{array}$ & $\begin{array}{l}\text { mildes ARDS: } \mathrm{P}_{\mathrm{a}} \mathrm{O}_{2} / \mathrm{F}_{\mathrm{i}} \mathrm{O}_{2}= \\
\text { 201-300 mm } \mathrm{mg} \text { bei PEEP } \geq 5 \mathrm{~cm} \mathrm{H} \mathrm{H}_{2}\end{array}$ \\
\hline \multirow[t]{2}{*}{$\begin{array}{l}\text { ARDS: } \mathrm{p}_{\mathrm{a}} \mathrm{O}_{2} / \mathrm{F}_{\mathrm{i}} \mathrm{O}_{2} \text {-Quotient } \\
<200 \mathrm{mmHg}\end{array}$} & $\begin{array}{l}\text { moderates ARDS: } \mathrm{P}_{\mathrm{a}} \mathrm{O}_{2} / \mathrm{F}_{\mathrm{i}} \mathrm{O}_{2}= \\
101-200 \mathrm{mmHg} \text { bei } \mathrm{PEEP} \geq 5 \mathrm{~cm} \mathrm{H} \mathrm{H}_{2} \mathrm{O}\end{array}$ \\
\hline & $\begin{array}{l}\text { schweres ARDS: } \mathrm{P}_{\mathrm{a}} \mathrm{O}_{2} / \mathrm{F}_{\mathrm{i}} \mathrm{O}_{2} \leq 100 \mathrm{mmHg} \\
\text { bei PEEP } \geq 5 \mathrm{~cm} \mathrm{H} \mathrm{H}_{2} \mathrm{O}\end{array}$ \\
\hline
\end{tabular}

digung der Lunge hervorrufen, gelten hohe Atemzugvolumina (Volutrauma), hohe Beatmungsdrücke (Barotrauma) und hohe Scherkräfte („driving pressure“). Stress, der durch diese 3 Faktoren auf die Lunge ausgeübt wird, sorgt für eine Entzündungsreaktion (Biotrauma), die die Ausbildung/Verstärkung des Lungenödems begünstigt. In Studien konnte bewiesen werden, dass die Reduktion des Atemzugsvolumens auf Werte von $\leq 6 \mathrm{ml} / \mathrm{kg}$ KG (IBW) zur einer Reduktion der Sterblichkeit führt [8, 9].

Einige Patienten weisen bei schwerem ARDS ein so stark verringertes belüftetes Lungenvolumen auf („,babylung“), dass selbst Atemzugvolumina von $\leq 6 \mathrm{ml} / \mathrm{kg} \mathrm{KG}$ (IBW) eine Lungenschädigung zur Folge haben [10]. In diesen Fällen kann eine weitere Reduktion des Atemzugvolumens auf $\leq 4 \mathrm{ml} / \mathrm{kg} \mathrm{KG}$ (IBW) teilweise in Kombination mit einer ECMO-Therapie sinnvoll sein (ultraprotektive Beatmung).

Atelektasen werden durch einen kontinuierlichen positiven Druck am Ende eines Beatmungszyklus (PEEP) behandelt. Die Verbesserung der Oxygenierung durch den PEEP konnte bereits Anfang der 1970er-Jahre gezeigt werden [11]. Bei der Frage nach dem richtigen PEEP gilt es die Balance zwischen maximaler Öffnung der verschlossenen Alveolen und den negativen Nebenwirkungen eines überhöhten PEEP zu finden. Dazu gehören vor allem ein gesteigerter intrathorakaler Druck mit Reduktion des Herz-Zeit-Volumens durch verminderten venösen Rückfluss zum Herzen und die Abflussstörung der Jugularvenen, was einen Anstieg des intrakraniellen Drucks nach sich ziehen kann.

Im klinischen Alltag haben sich 2 Verfahren des „best peep trial“ als praktikabel erwiesen: Bei stabilen hämodynamischen Parametern (Blutdruck, Herzfrequenz) wird entweder ansteigend von niedrigen Werten bzw. abfal- 


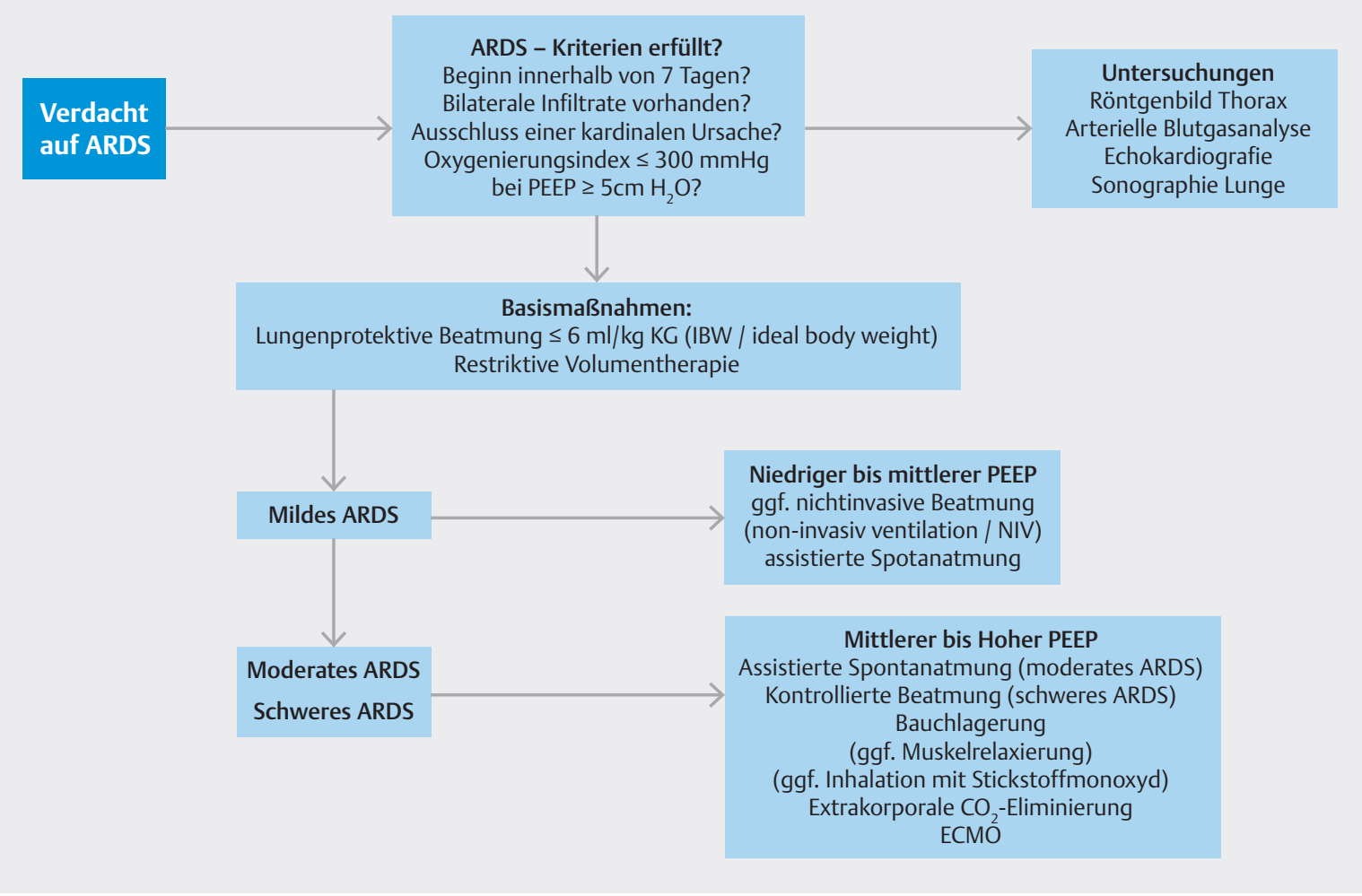

Abb. 1 Flussdiagramm: symptombezogene Behandlungsstrategie des ARDS.

lend von höheren Werten der PEEP variiert und die Veränderung der Oxygenierung gemessen. Der beste PEEP ist derjenige, bei dem die folgende ab- oder aufsteigende Veränderung keine Verbesserung der Oxygenierung erzielt [12]. Ist die Beatmung anhand der Vorgaben optimal eingestellt ( $\triangleright$ Tab.3), werden diese Einstellungen auch für den Intensivtransport zunächst übernommen. Bei Wechsel des Respirators ist ein PEEP-Verlust unbedingt zu vermeiden, da dieser zur erneuten Aktelektasenbildung führen kann. Von der Diskonnektion bis zur Rekonnektion wird der Tubus mittels einer Klemme verschlossen.

\section{Muskelrelaxierung}

Ob eine Muskelrelaxierung im Rahmen der maschinellen Beatmung einen Vorteil bringt, ist bis heute nicht abschließend geklärt. Ein Vorteil konnte nicht zweifelsfrei erwiesen werden, jedoch gibt es Hinweise, dass bei einem leichten bis mittelschweren ARDS eine frühzeitig ermöglichte maschinell unterstütze Spontanatmung (innerhalb der ersten 48 Stunden nach Intubation) unter anderem die $\mathrm{CO}_{2}$-Elimination sowie die Oxygenierung verbessert. Des Weiteren bleibt die Zwerchfellaktivität erhalten, was einen Vorteil beim Weaning darstellt.

Untersuchungen im Bereich des schweren ARDS ergaben einen Überlebensvorteil einer Relaxierung innerhalb der ersten 48 Stunden. Die Autoren der aktuellen S3-Leitlinie
„Invasive Beatmung und Einsatz extrakorporaler Verfahren bei akuter respiratorischer Insuffizienz" sehen bei diesen Studien jedoch erhebliche methodische Mängel. Eine Empfehlung wird daher lediglich für die kontrollierte Beatmung im Gegensatz zur assistierten Spontanatmung beim leichten und mittelschweren ARDS gegeben. Die Relaxierung bleibt eine Einzelfallentscheidung.

\section{Bauchlagerung}

Die durch ein ARDS hervorgerufenen Veränderungen in der Lunge sind nicht homogen. Mittels Computertomografie konnte dies bei Patienten in Rückenlage dargestellt werden. Während die dorsalen Lungenabschnitte ein ausgeprägtes Lungenödem und Atelektasen zeigen, weisen ventrale Abschnitte teilweise noch intakte Lungenbereiche auf [13]. Gemäß dem 3-Zonen-Modell nach West ist die Lungendurchblutung in den unteren Abschnitten am höchsten. Diese Bereiche nehmen jedoch aufgrund ausgeprägter Veränderungen nicht am Gasaustausch teil und sorgen somit dafür, dass nichtoxygeniertes Blut in den Organismus gelangt (Rechts-Links-Shunt).

Dass eine Bauchlagerung eine Verbesserung des Gasaustausches bewirkt, ist schon seit den 1970er-Jahren bekannt, jedoch konnten die genauen Gründe erst viel später ermittelt werden. Es kommt unter anderem zu einer verbesserten Verteilung der Atemgase, der Lungendurchblutung und Atemmechanik. Dieser Effekt setzt 
bereits nach kurzer Zeit ein und ist abhängig von der Dauer. Ein Intervall von 16 Stunden sollte dabei möglichst nicht unterschritten werden [14].

\section{Stickstoffmonoxyd}

Inhalatives Stickstoffmonoxyd führt selektiv zu einer verbesserten Durchblutung von Lungenarealen, die gut belüftet sind. Die sich aus diesem Effekt ergebende Abnahme des Rechts-Links-Shunts hält jedoch nur ca. 24-48 Stunden an und hat keine Auswirkung auf das Überleben des Patienten. Der Verdacht, dass dieses Verfahren zu einer Nierenfunktionsstörung führen könnte, lässt es lediglich als Notfallverfahren zu, um Zeit zu gewinnen, bis eine andere Therapie greift [15].

\section{Extrakorporale Membranoxygenierung (ECMO)}

Die ECMO ist ein Verfahren, das die Lungenfunktion vollständig ersetzen kann. Über 2 großlumige Kanülen wird das Blut des Patienten mittels Zentrifugalpumpe zu einem Membranoxygenator geleitet ( $\boldsymbol{A} \mathbf{A b} \mathbf{b} . \mathbf{2})$. Dort findet über eine semipermeable Membran der Gasaustausch statt. Ziel dieses Verfahrens ist es, die Lungenfunktion so lange zu ersetzen, bis es zu einer Organerholung kommt („bridge to recovery“). Dieses Verfahren wird bei schwerem ARDS erst dann empfohlen, wenn unter Ausschöpfung aller anderen Maßnahmen die Hypoxämie $\left(\mathrm{p}_{\mathrm{a}} \mathrm{O}_{2} / \mathrm{F}_{\mathrm{i}} \mathrm{O}_{2}\right.$-Quotient $\left.<80 \mathrm{mmHg}\right)$ weiter besteht.

\section{KERNAUSSAGEN}

- Das ARDS ist eine Erkrankung mit einer schwersten Gasaustauschstörung.

- Pathophysiologisch führen die Veränderungen in der Lunge zu einer Verringerung der Gasaustauschfläche und einer erhöhten Diffusionsstrecke.

- Seit 2012 wird das ARDS gemäß Berlin-Klassifikation in 3 Schweregrade eingeteilt.

- Neben der kausalen Therapie spielen vor allem die symptombezogenen Maßnahmen eine entscheidende Rolle in der Therapie.

- Die lungenprotektive Beatmung, in Kombination mit einer Bauchlagerung, ist der wichtigste symptombezogene Therapieansatz.

- Eine ECMO-Therapie wird nur empfohlen, wenn eine Hypoxämie nach Ausschöpfung aller anderen Maßnahmen weiter besteht.
- Tab. 3 Empfohlene Beatmungseinstellung bei Patienten mit respiratorischer Insuffizienz gemäß S3-Leitlinie „Invasive Beatmung und Einsatz extrakorporaler Verfahren bei akuter respiratorischer Insuffizienz“, Stand vom 04.12.2017.

\begin{tabular}{|l|l|}
\hline Beatmungsparameter & Einstellung \\
\hline Tidalvolumen $\left(\mathrm{V}_{\mathrm{t}}\right)$ & $\leq 6 \mathrm{ml} / \mathrm{kg} \mathrm{KG}(\mathrm{IBW})$ \\
\hline PEEP & $\begin{array}{l}\text { mind. } \geq 5 \mathrm{~cm} \mathrm{H} \mathrm{H}_{2} \mathrm{O} \text { bzW. „best peep trial“ } \\
\text { durchführen }\end{array}$ \\
\hline endinspiratorischer Druck $\left(\mathrm{P}_{\max }\right)$ & $<30 \mathrm{~cm} \mathrm{H}_{2} \mathrm{O}$ \\
\hline "driving pressure“ & $\leq 15 \mathrm{~cm} \mathrm{H}_{2} \mathrm{O}$ (Differenz: $\left.\mathrm{P}_{\max }-\mathrm{PEEP}\right)$ \\
\hline I:E-Verhältnis & $1: 1$ bis $1: 1,5$ \\
\hline $\mathrm{F}_{\mathrm{i}} \mathrm{O}_{2}$ & so niedrig wie möglich; Ziel $\mathrm{P}_{\mathrm{a}} \mathrm{O}_{2}$ von \\
& $60-80 \mathrm{mmHg}$ \\
\hline
\end{tabular}

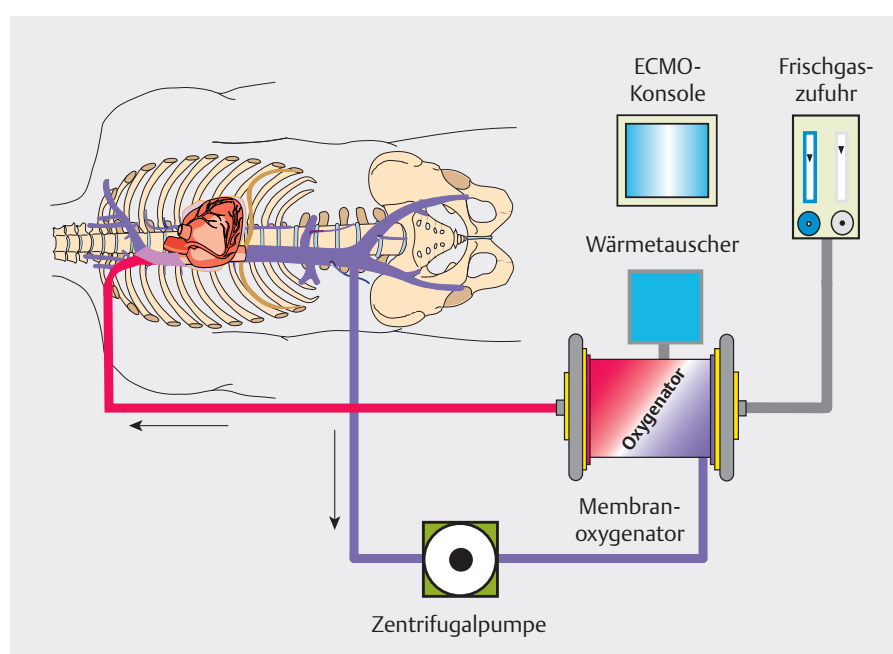

- Abb.2 Aufbau und Funktionsweise der ECMO. Quelle: Oczenski W. Aufbau und Funktionsprinzip. In: Oczenski W, Hrsg. Atmen - Atemhilfen. 10. Aufl. Stuttgart: Thieme; 2017. doi:10.1055/b-004-140689.

\section{Interessenkonflikt}

\section{Erklärung zu finanziellen Interessen}

Forschungsförderung erhalten: nein; Honorar/geldwerten Vorteil für Referententätigkeit erhalten: nein; Bezahlter Berater/interner Schulungsreferent/Gehaltsempfänger: nein; Patent/Geschäftsanteile/Aktien (Autor/Partner, Ehepartner, Kinder) an im Bereich der Medizin aktiven Firma: nein. Patent/ Geschäftsanteile/Aktien (Autor/Partner, Ehepartner, Kinder) an zu Sponsoren dieser Fortbildung bzw. durch die Fortbildung in ihren Geschäftsinteressen berührten Firma: nein.

Erklärung zu nichtfinanziellen Interessen

Die Autorinnen/Autoren geben an, dass kein Interessenkonflikt besteht 
Autorinnen/Autoren

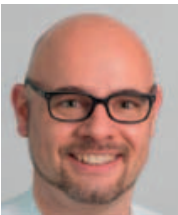

\section{Michael Gores}

Facharzt für Anästhesiologie, Zusatzbezeichnung Intensiv- und Notfallmedizin, Qualifikation LNA/ÄLRD, DEGUM I Anästhesiologie, PHTLS-Provider. Michael Gores ist tätig als Oberarzt in der Abteilung für Anästhesiologie und Intensivmedizin am Evangelischen Krankenhaus Bergisch Gladbach.

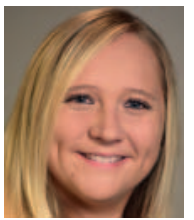

\section{Jennifer Schneck}

2011-2012 RAiP, 2012-2017 Rettungsassistentin. Jennifer Schneck ist Notfallsanitäterin und Praxisanleiterin beim Malteser Hilfsdienst e. V. Leverkusen und seit Oktober 2018 Lehrkraft am Malteser Bildungszentrum Rheinland.

\section{Korrespondenzadresse}

\section{Michael Gores}

Abteilung für Anästhesie und Intensivmedizin, Evangelisches Krankenhaus Bergisch Gladbach Ferrenbergstraße 24

51465 Bergisch Gladbach

m.gores@evk.de

\section{Literatur}

[1] Goddemeier C. Geschichte der Medizin: Erfinder des Stethoskops. Dtsch Arztebl International 2006; 103: 1436

[2] Laennec RTH, Forbes JS. A treatise on the diseases of the chest: in which they are described according to their anatomical characters, and their diagnosis established on a new principle by means of acoustick instruments: with plates. London: T. and G. Underwood; 1821

[3] Ashbaugh DG, Bigelow DB, Petty TL et al. Acute respiratory distress in adults. Lancet 1967; 2: 319-323

[4] Petty TL. In the cards was ARDS: how we discovered the acute respiratory distress syndrome. Am J Respir Crit Care Med 2001; 163: 602-603
[5] Bernard GR, Artigas A, Brigham KL et al. The American-European Consensus Conference on ARDS. Definitions, mechanisms, relevant outcomes, and clinical trial coordination. Am I Respir Crit Care Med 1994; 149: 818-824

[6] Force ADT, Ranieri VM, Rubenfeld GD et al. Acute respiratory distress syndrome: the Berlin Definition. JAMA 2012; 307: 2526-2533

[7] Walkey A], Summer R, Ho V et al. Acute respiratory distress syndrome: epidemiology and management approaches. Clin Epidemiol 2012; 4: 159-169

[8] Brower RG, Matthay MA, Morris A et al. Ventilation with lower tidal volumes as compared with traditional tidal volumes for acute lung injury and the acute respiratory distress syndrome. N Engl J Med 2000; 342: 1301-1308

[9] Amato MB, Barbas CS, Medeiros DM et al. Effect of a protective-ventilation strategy on mortality in the acute respiratory distress syndrome. N Engl J Med 1998; 338: 347-354

[10] Terragni PP, Rosboch G, Tealdi A et al. Tidal hyperinflation during low tidal volume ventilation in acute respiratory distress syndrome. Am J Respir Crit Care Med 2007; 175: 160166

[11] Falke KJ, Pontoppidan H, Kumar A et al. Ventilation with endexpiratory pressure in acute lung disease. J Clin Invest 1972; 51: $2315-2323$

[12] Oczenski W. Atmen - Atemhilfen. 8. Aufl. Stuttgart: Thieme; 2008

[13] Sheard S, Rao P, Devaraj A. Imaging of acute respiratory distress syndrome. Respir Care 2012; 57: 607-612

[14] Fridrich P, Krafft $P$, Hochleuthner $\mathrm{H}$ et al. The effects of longterm prone positioning in patients with trauma-induced adult respiratory distress syndrome. Anesth Analg 1996; 83: 12061211

[15] Gebistorf F, Karam O, Wetterslev ] et al. Inhaled nitric oxide for acute respiratory distress syndrome (ARDS) in children and adults. Cochrane Database Syst Rev 2016: CD002787

Bibliografie

DOI https://doi.org/10.1055/a-0646-6938

retten 2019; 8: 347-354

(c) Georg Thieme Verlag KG, Stuttgart · New York ISSN 2193-2387 


\section{Punkte sammeln auf CMEthieme.de}

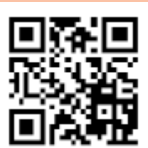

Diese Fortbildungseinheit ist in der Regel 12 Monate online für die Teilnahme verfügbar.

Den genauen Einsendeschluss finden Sie unter https://eref.thieme.de/CXB4KA6.

Sollten Sie Fragen zur Online-Teilnahme haben, finden Sie unter https://cme.thieme.de/hilfe

eine ausführliche Anleitung. Wir wünschen viel Erfolg beim Beantworten

der Fragen!

Unter https://eref.thieme.de/CXB4KA6 oder über den QR-Code kommen Sie direkt zur Startseite des Wissenstests.

\section{Frage 1}

Die Sterblichkeit infolge ARDS liegt bei ...
A mehr als $90 \%$.
B mehr als $60 \%$.
C mehr als $70 \%$.
D mehr als $40 \%$.
E unter $10 \%$.

\section{Frage 2}

Was macht man im wörtlichen Sinn mit dem Stethoskop?
A das „Herz abhören“
B die "Lunge betrachten“
C den „Brustkorb abhören“
D den „Brustkorb betrachten“
E die „Lunge abhören“

\section{Frage 3}

Was zählt nicht zur indirekten Schädigung der Lunge?
A Sepsis (nicht von der Lunge ausgehend)
B Pankreatitis
C Massivtransfusion
D schweres Trauma
E Aspiration

\section{Frage 4}

Was ist ein Kriterium des ARDS?

A Oxygenierungsindex $\leq 300 \mathrm{mmHg}$ bei PEEP $\leq 5 \mathrm{~cm} \mathrm{H}_{2} \mathrm{O}$

B bilaterale diffuse Infiltrate

C kardiales Lungenödem

D Beginn > 14 Tage

E Infektwerte im Labor sind immer unauffällig

\section{Frage 5}

Wie hoch ist das empfohlene Atemzugvolumen bei Patienten mit ARDS?
A $\quad 6-8 \mathrm{ml} / \mathrm{kg} \mathrm{KG}$ (IBW)
B $\quad 10-12 \mathrm{ml} / \mathrm{kg} \mathrm{KG}$ (IBW)
$C \leq 6 \mathrm{ml} / \mathrm{kg}$ KG (aktuelles Körpergewicht)
D Eine Empfehlung hierzu existiert nicht.
$\mathrm{E} \leq 6 \mathrm{ml} / \mathrm{kg} \mathrm{KG}(\mathrm{IBW})$

\section{Frage 6}

Welche dieser Aussagen ist richtig?

A Im 3-Zonen-Modell nach West ist die Lungendurchblutung basal am geringsten.

$B$ Es sollte ein $\mathrm{P}_{\mathrm{a}} \mathrm{O}_{2}$ von mindestens $110 \mathrm{mmHg}$ angestrebt werden.

C Surfactant wird durch die Pneumozyten Typ I gebildet

D Die Nettodiffusion ist abhängig von der Diffusionstrecke und der Austauschfläche.

E Der PEEP wird immer anhand einer komplexen Formel berechnet.

\section{Frage 7}

Welche dieser Aussagen ist falsch?

A Die Berlin-Klassifikation entstand 2012.

B Folge des PEEP kann ein gesteigerter intrakranieller Druck sein.

C Die Bauchlagerung sollte 16 Stunden am Stück nicht überschreiten.

D Die Formel des Oxygenierungsindex lautet: $\mathrm{P}_{\mathrm{a}} \mathrm{O}_{2}(\mathrm{mmHg}) / \mathrm{FiO}_{2}$

E Eine generelle Empfehlung zur Muskelrelaxierung besteht nicht.

\section{Frage 8}

Was ist nicht verantwortlich für die Gasaustauschstörung beim ARDS?

A reduzierte Bildung von Surfactant

B Lungenödem mit erhöhter Diffusionsstrecke

C erhöhter Rechts-Links-Shunt

D verringerte Austauschfläche der Lunge

E kardiales Lungenödem

\section{Frage 9}

Welche Aussage zur Behandlung des ARDS ist richtig?

A Eine Relaxierung ist eine Einzelfallentscheidung.

B Der Effekt von Stickstoffmonoxid nimmt mit der Länge der Behandlung stetig zu.

C Die Spontanatmung sollte vor allem bei einem schweren ARDS angestrebt werden.

D Das $\mathrm{FiO}_{2}$ sollte so hoch gewählt werden, dass ein Sauerstoffpartialdruck im Blut von mindestens $100 \mathrm{mmHg}$ erreicht wird.

E Surfactant fördert eine Atelektasenbildung.

- Weitere Fragen auf der folgenden Seite... 


\section{Punkte sammeln auf CME.thieme.de}

Frage 10

Welche Aussage trifft zu?

A Die Entzündungsreaktion beim ARDS beschränkt sich auf die Alveolen.

B Ein funktionierender Euler-Liljestrand-Mechanismus verschlechtert das ARDS.

C Ein Ziel der Beatmungseinstellung ist die Vermeidung eines Barotraumas.

D Kriterium für ein schweres ARDS ist $\mathrm{P}_{\mathrm{a}} \mathrm{O}_{2} / \mathrm{F}_{\mathrm{i}} \mathrm{O}_{2} \leq 150 \mathrm{mmHg}$.

E Eine ECMO-Therapie wird sehr frühzeitig empfohlen. 